\title{
Derivation Factors of Smart Space Awareness for Effective CPTED
}

\author{
Donghyok Suh and Jeonghwa Song, \\ Namseoul University, 97, Daehak-Ro, Senghwaneub and Seobuk-Ku, \\ 32588 Cheonan Chungnam, Korea \\ \{absuh,songjh\}@nsu.ac.kr
}

\begin{abstract}
Crime prevention activities that take place in the physical environment, such as police patrolling, can work effectively when they are combined with intelligent detection that uses information technology (IT). Many different real-world factors must be considered when implementing intelligent detection methods. In risky real-world situations, fixed factors, static factors, and a variety of dynamic factors all play important roles. This study aimed to determine the variable factors involved in risky situations by performing an analysis on data from specific crime incidents. Factors related to the occurrence of the specific crime were determined and risk of occurrence was calculated using these factors with a multi-factor convergence scheme. The analysis and risk calculation performed in this study are expected to contribute to the development of a more efficient and intellectualized prevention service, as these can help provide information about real-time risk levels for particular public safety situations.
\end{abstract}

Keywords: crime prevention, data convergence, situation deduction.

\section{Introduction}

Research is currently being done on the reduction of crime occurrence through improvement of the urban structure and environment. Many studies focus on intelligent crime detection as an important method for restricting the occurrence of crime. One other way to reduce crime is to improve residential urban environments so that these regions facilitate less crime $[1,2,3,4]$. Crime may be reduced by simply improving the physical environment using measures such as purification of the environment in crime-ridden districts, improvement of unsanitary facilities, and the installation of surveillance cameras [5, 6, 7]. Unfortunately, it is often easy for criminals to detect and avoid common security measures. Violent offenders tend to create extensive plans to avoid being caught, and as such, techniques for evading or bypassing various crime prevention methods are becoming more and more advanced. Most existing security systems designed for crime prevention include surveillance cameras and emergency alarms $[8,9,10]$. While these measures may put some pressure on criminals, they are also relatively easy to evade. Additionally, even though surveillance cameras can provide useful information that helps identify and arrest criminals after the crime has already occurred, the crime prevention effects of surveillance camera use are controversial. Though prevention is important for all crimes, it is particularly important in the case of violent crime. Once a violent crime has taken place, the damage has already been done, regardless of whether the culprit is found and arrested or not. The tremendous harm caused by violent crime can never be undone; thus, more effective and efficient measures are required for the prevention of these crimes. In the past, measures have been taken to improve physical spaces with high levels of crime. Such measures have included painting murals on alley walls in the downtown region and installing flower beds. Surveillance cameras have also been installed, though the preventative effects of these cameras are still ambiguous and 
controversial. This is why electric detection facilities should be involved in the redesign of the physical spaces where violent crime occurs most. A security scheme including both an intelligent detection function and information technology (IT) can overcome ambiguity and strengthen the performance of existing systems [21,23].

It is important to understand the risk factors that must be perceived by the sensors and network system when preparing an intelligent detection system that can detect crime occurrence risk in urban spaces. More effective and efficient crime prevention can be expected when intelligent detection functions that include factors for computing risk level are integrated with the facilities of high-risk physical environments.

Safety Map, a security application developed and used by the Korean National Police Agency, is an example of an intelligent detection system for crime prevention. The Korean National Police Agency determines the risk of crime occurring in a certain area based on crime occurrence data collected by the application. The data is then analyzed and police presence, including the number of patrolling officers, is increased in areas with higher crime risk. Safety Map has helped reduce crime rate and increase the number of criminal arrests made in high-risk areas [5,7,22,24].

Crime prevention strategies can be improved when crime prevention activities are implemented in conjunction with intelligent detection methods that make use of IT. Research done by the Korean National Police Agency using crime data analysis technology has mainly focused on determining crime occurrence risk for segmented areas based on the crime incident data from each respective area. Though this research has been helpful, the research techniques used to study crime occurrence risk must be developed further in order to gain more insight into this topic. The prediction of regional crime occurrence risk is currently based on incident data from the past, such as data about areas and times with previous histories of crime occurrence. Thus, many dynamic real-world factors that may also play roles in crime occurrence have not been addressed by the current research methods. For example, the crime rate may decrease in a once crimeridden district following an increase in floating population. Similarly, a previously lowcrime area may see an increase in the likelihood of crime occurrence following a decrease in floating population, and some pedestrians may experience unstable conditions due to drinking, etc $[11,13,15]$.

The following are real cases of violent crimes. In 2012, a woman in her early 20s was standing alone in front of a subway station at five am, at which time she was kidnapped. In a similar incident in Cheonan-si in 2015, an inebriated woman was walking alone and was sexually assaulted by two men. These two incidents share one thing in common: they both took place in areas with relatively low crime occurrences. Similar incidents of this nature had not occurred in the past in either of these areas. Clearly, there were different factors that played roles in the occurrences of these specific crimes. For example, if there had been other people present during the first incident or if the victim had not been drunk during the second incident, the outcomes may have been different $[18,19]$. 


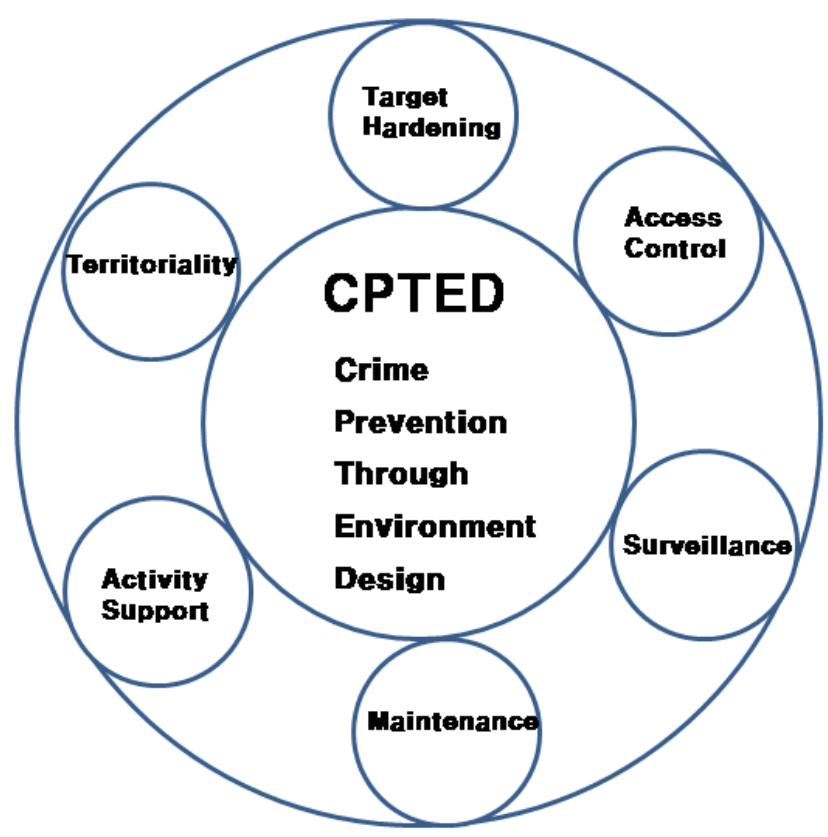

Figure 1. CPTED (Crime Prevention Through Environment Design)

Intelligent detection methods are thus necessary for crime prevention, as they can be used for environmental improvement and the identification of various dynamic factors related to each individual incident, in addition to identification of the fixed factors that should be included in intelligent crime detection[12,16].

This study focused on predetermining the dynamic factors related to specific high-risk situations by analyzing specific incidents of violent crime. More specifically, the crimes analyzed for the purpose of this study were roadside kidnapping and sexual assault cases occurring in Cheonan-si, Chungcheongnam-do, Korea. Cheonan-si is an industrial city with a population of 0.6 million. The city has shown a marked increase in sexual crimes over the past five years. The present study analyzed these incidents and extracted potential environmental and dynamic factors that may have had an impact on the occurrences of these incidents. This study also aimed to determine which sensors could detect these factors in daily life, and based on these results, a scheme was proposed for calculating the risk levels for violent crimes using the values detected and reported by each sensor. This kind of calculation scheme can help simplify the process of obtaining information by evaluating different trends in the signals detected by the sensors over time. The information obtained from the changing values detected by the sensors can be used for the calculation of real-time crime risk, thus resulting in the possibility of prompt responses to high-risk situations.

This paper is organized as follows. Following the Introduction, Chapter 1 and Chapter 2 summarize the related literature. Chapter 3 reports on the use of an actual incident analysis to determine dynamic factors related to that specific incident. Chapter 4 determines which sensors can detect the dynamic factors determined in Chapter 3 in a real-world situation, and these sensors are used to calculate the risk-level indicated by each factor and to investigate the effectiveness. Chapter 5 presents an evaluation of the study and the conclusion. 


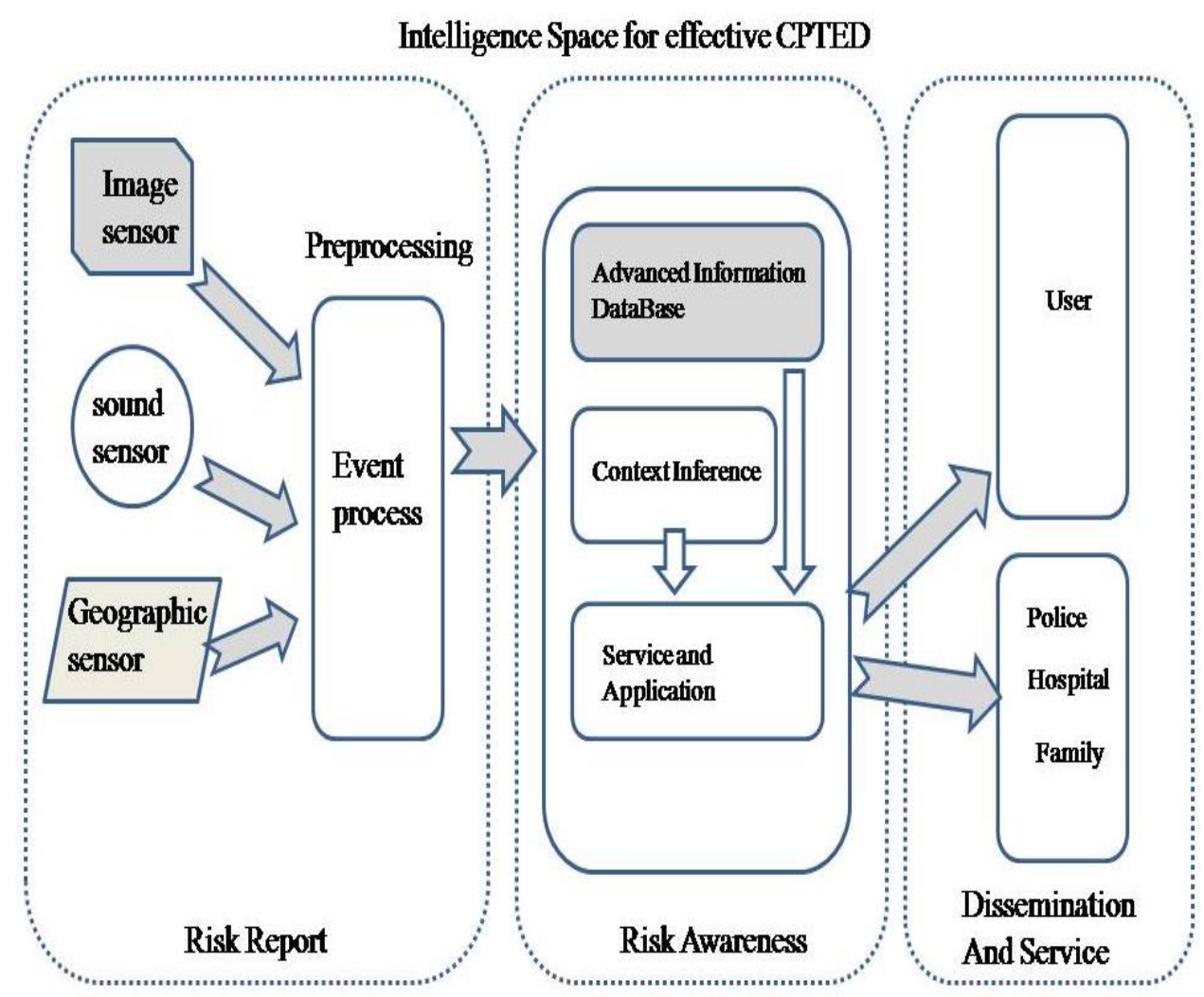

Figure 2. Intelligence Space for Effective CPTED

Figure 2 shows Intelligence space for effective CPTED. Intelligent detection methods are thus necessary for crime prevention. At first, datasets from each sensor should be passed through the course of event preprocessing. Then advanced information database provides a model of each context which is already known. But advanced information database cannot provide it for the unknown situation. In this case, context inference course can estimate and evaluate the unknown situation for risk awareness. Finally, the estimated degree of risk is sent to users.

\section{Determining Risk Factors and Calculating Risk to Public Safety}

This study aims to find the dynamic factors that variably increase or decrease the risk level of crime occurrence in a specific condition or environment. It also aims to determine, based on existing statistical analyses of public safety and crime prevention data, which static factors affect crime occurrence. The study also attempts to calculate the real-time risk of crime occurrence for each incident based on these variable risk factors. The use of a real-time risk calculation should provide better security and more effective risk prevention than existing methods of risk assessment.

\subsection{Necessity of Finding Dynamic Factors}

According to the Crime Occurrence Statistics (2012) collected by the Korean National Police Agency, "mutual acquaintance" is the most common relationship type between assailant and victim in both murder and rape cases. However, incidents of such crimes being perpetrated by strangers also account for a considerable proportion of these crimes. This study focused on one type of violent incident involving ordinary citizens who have no relationship with or connection to their attackers - that is, it focuses on the kidnapping 
and sexual assault of women. These attacks occurred when the victims were performing routine daily activities, such as commuting. Many of these incidents occurred in places that are not classified as high-crime areas. Just as a high-accident area is not necessarily susceptible to accidents 24 hours a day, 365 days a year, safe areas are not necessarily always safe. In other words, the risk of crime occurrence can decrease in high-crime areas if the conditions are not conducive to committing a crime, while crime can also occur in safe areas if certain factors play a role. Hence, to understand risk level in terms of the role it can play in crime prevention, risk should be calculated in a more advanced manner by determining the dynamic factors related to crime occurrence.

\subsection{Extraction of Dynamic Factors}

Risk factors that either increase or decrease the risk of crime occurring over time are defined as dynamic factors. Time itself is one example of a dynamic factor. This study analyzed cases of sexual assault and sexual harassment committed by strangers that occurred in Cheonan-si and the surrounding area. Three dynamic factors were found to play roles in the occurrence of sexual assault and sexual harassment in these areas: floating population, time, and spatial characteristics.

1) Floating population: The existence of a floating population where the victim resides, as well as the size of that floating population, seems to be related to the occurrence of sexual harassment and assault. The cases below are all examples of incidents that occurred in an area with a very small floating population. It should also be noted that none of these incidents occurred in typically crime-ridden districts.

- Case 1-01) September 8, 2013-Near a Ministop convenience store, Giro-li, Yijangmyeon, Cheonan-si, Chungcheongnam-do, Korea

- Case 1-02) October 12, 2013 - On the sidewalk of a parking lot at Gongju University, Budae-dong, Seobuk-gu, Cheonan-si, Chungcheongnam-do, Korea

- Case 1-03) March 25, 2014-In front of Baekseok University, Anseo-dong, Dongnam-gu, Cheonan-si, Chungcheongnam-do, Korea

- Case 1-04) August 16, 2013-In front of a 7-Eleven convenience store, Jiksan-eup, Sameun-li, Cheonan-si, Chungcheongnam-do, Korea Seobuk-gu

- Case 1-05) June 19, 2013-In the Shinjeonho recreational park outside a Onjeoncheong UL City building, Asan-si, Chungcheongnam-do, Korea

- Case 1-06) July 10, 2010 - On a pedestrian overpass staircase near Daewoo Apartment, Dujeong-dong, Seobuk-gu, Cheonan-si, Chungcheongnam-do, Korea

- Case 1-07) July 27, 2010-In front of a GS25 convenience store, Dujeong-dong, Seobuk-gu, Cheonan-si, Chungcheongnam-do, Korea

- Case 1-08) July 11, 2013-In front of the KCC Sawon Apartment, Yeomchi-eup, Seowon-li, Asan-si, Chungcheongnam-do, Korea

- Case 1-09) January 5, 2012 - In front of a Mr. K Stationery Store, Sunghwan-eup, Sunghwan-li, Cheonan-si, Chungcheongnam-do, Korea Seobuk-gu

- Case 1-10) March 22, 2013-On Stone Wall road, between Doongji Daycare Center and Heungjin Construction, Yooryang-dong, Dongnam-gu, Cheonan-si, Chungcheongnam-do, Korea

- $\quad$ Case 1-11) June 9, 2013 - In front of Daga Daycare Center, Daga-dong, Cheonan-si, Chungcheongnam-do, Korea

Most of the above incidents occurred in places that periodically experience drastic drops in floating population. Although these places were all open to the public and were perceived as being relatively safe, the few members of the nearby floating populations made these places more susceptible to crime. 
2) Time: In the cases listed below, time is suspected to have played a certain role in the occurrence of the crime, despite the crime occurring in what was considered to be a relatively safe area.

- Case 2-01) December 21, 2012-At 00:10, at Panjeong intersection, Jiksan-eup, Seobuk-gu Cheonan-si, Chungcheongnam-do, Korea

- Case 2-02) June 6, 2013-At 01:20, on a road near Feelhouse, Dujeong-dong, Seobuk-gu, Cheonan-si, Chungcheongnam-do, Korea

- Case 2-03) June 15, 2013-At 23:05, between the Dujeong Station and Ian Apartment, Buseong-dong, Seobuk-gu, Cheonan-si, Chungcheongnam-do, Korea

- Case 2-04) July 11, 2013-At 04:00, at Wooseong Foods, Seongjeong-dong, Seobuk-gu, Cheonan-si, Chungcheongnam-do, Korea

- Case 2-05) September 8, 2013-At 02:00, near a Ministop convenience store, Giro-li, Yijang-myeon, Seobuk-gu, Cheonan-si, Chungcheongnam-do, Korea

- Case 2-06) September 21, 2013-At 03:10, at Withhouse Club, Dujeong-dong, Seobuk-gu, Cheonan-si, Chungcheongnam-do, Korea

- Case 2-07) June 22, 2012-At 23:50, on the street behind Goodmorning Orthopedics, Dujeong-dong, Seobuk-gu, Cheonan-si, Chungcheongnam-do, Korea

- Case 2-08) July 26, 2012-At 01:50, on the road in front of Neulbom Restaurant, Daeheung-dong, Dongnam-gu, Cheonan-si, Chungcheongnam-do, Korea

- Case 2-09) August 4, 2012-At 04:30, on a staircase in Cheonan Station, Daeheungdong, Dongnam-gu, Cheonan-si, Chungcheongnam-do, Korea

- Case 2-10) September 14, 2013-At 04:40, on the road in front of Jin Orthopedics, Cheongsu-dong, Dongnam-gu, Cheonan-si, Chungcheongnam-do, Korea

- Case 2-11) May 20, 2010-At 23:00, on the Gongsu underground roadway, Baebang-eup, Gongsu-li, Asan-si, Chungcheongnam-do, Korea

In many of the above cases, the incident took place near a main road or in a public place. Although the spatial characteristics of these locations indicates that the probability of a crime occurring should be low, these places became more susceptible to crime late at night. Changes in time thus played a role in the rate of crime occurrence.

3) Spatial characteristics: One of the factors observed in some of the cases was that the victims became targets when they moved from a wide road to a narrow road. In the cases listed below, the victims moved from a brightly lit area on a wide road to a poorly lit area on a narrow road.

- Case 3-1) August 13, 2013-In the alley near Saemannam Church, Bongmyungdong, Dongnam-gu, Cheonan-si, Chungcheongnam-do, Korea

- Case 3-2) July 6, 2010-In the alley beside Hoseo Wedding Hall, Baebang-eup, Segyo-li, Asan-si, Chungcheongnam-do, Korea

- Case 3-3) July 16, 2010 - In front of Doojeong Housing, Dujeong-dong, Seobuk-gu, Cheonan-si, Chungcheongnam-do, Korea

These incidents all share one common feature: in every case, the victim was spotted by the perpetrator in the main road before she moved to a narrow alley, where the perpetrator then chased her to commit the crime. Hence, it is necessary to consider the moving path of the victim as a factor that may influence the occurrence of sexual assault or harassment. Although other crime-related factors can also be extracted in more specified studies in the future, this study was primarily focused on the extraction of representative dynamic factors and the role these factors may play in schemes for determining the numerical calculation of risk. 


\section{Real-time Risk Calculation and Evaluation}

This chapter determines the sensors that can be used to detect the dynamic factors that were extracted in Chapter 3. The risk values are then calculated using the measures taken by these sensors. Through this process, the validity and necessity of the extracted dynamic factors are proven. Factors influencing the risk of roadside crime occurrence are calculated considering the following main factors: (1) floating population, (2) time, and (3) spatial characteristics.

The next section outlines the actual scheme used to calculate risk based on the previously extracted variable risk factors.

Risk factor 1: Nearby floating population

Members of the floating population were detected with infrared sensors placed in the moving paths of pedestrians.

The following case assumes that $n$ infrared sensors are installed in nearby area S, among which $\mathrm{m}$ infrared sensors detect objects (vehicles, walking floating population members, etc.). First, the fundamental risk level at the current time is assigned as

$$
\alpha_{\mathrm{t}}=1-\frac{m}{s_{n}}
$$

Risk level decreases when there are more detected infrared sensors per unit area. According to the increases and decreases in risk level, the following computation was implemented. Each set of data detected by the infrared sensor in unit area $\mathrm{S}$ at time $\mathrm{t}$ was defined as:

$$
\mathrm{S}_{\mathrm{t}}=<a_{1}, a_{2}, a_{3}, \cdots, a_{m}>
$$

Based on the method suggested by Deypir, Hadi Sadreddini, and Hashemi (2012), which emphasized a method "towards a variable size sliding window model for frequent item set mining over data streams," a change of frequent items set was defined as:

$$
F_{\text {Change }}\left(T^{\prime}\right)=\frac{\left|F_{T}^{+}\left(T^{\prime}\right)\right|+\left|F_{T}^{-}\left(T^{\prime}\right)\right|}{\left|F_{T}\right|+\left|F_{T}^{+}\left(T^{\prime}\right)\right|}
$$

Here, the newly received data area was defined as:

$$
S_{t}^{+}=S_{t}-S_{t-1}
$$

Data that were no longer received in the next time period were defined as:

$$
S_{t}^{-}=S_{t-1}-S_{t}
$$

Changes in the data that were detected and reported by the infrared sensors were defined as follows:

$$
S C_{t}=\frac{n\left(S_{t}^{+}\right)+n\left(S_{t}^{-}\right)}{n\left(S_{t}\right)+n\left(S_{t}^{+}\right)}
$$

The equation above reflects the changes in the nearby floating population. The calculated results have values within the range of $[0,1]$. These values became close to zero considering the region with the larger floating population.

Following these calculations, the fundamental risk levels established earlier were multiplied. This was done to take into account both the fundamental risk level and the 
changes in floating population. The value of the floating population factor was defined as follows:

$$
P(t)=\alpha_{t} S C_{t}
$$

\section{Risk factor 2: Time}

The statistical frequency of the incidents was considered according to time period. The times for each occurrence were as follows:

$$
\begin{aligned}
& \text { Late night (00:00-04:00)-0.358957 } \\
& \text { Dawn (04:00-07:00)-0.119218 } \\
& \text { Morning (07:00-12:00)-0.104719 } \\
& \text { Afternoon (12:00-18:00)-0.121600 } \\
& \text { Early evening (18:00-20:00)-0.134298 } \\
& \text { Night (20:00-24:00)-0.161209 }
\end{aligned}
$$

The values given above indicate the frequency of incidents occurring during each time period, rather than the usual values, which involve using a simple sum of occurrence numbers. The above values were calculated as follows:

Frequency by time $=$ Number of incidents $/$ Length of time

The function of the occurrence time variable can be estimated based on the values above.

The graph above illustrates the time-frequency estimates. The $\mathrm{x}$-axis indicates 24 hours and the y-axis indicates the frequency of occurrence. The time factors can be expressed in the periodic function of the graph above. That is, the time factors can be expressed as a single value of the periodic function, as in the graph above. For the convenience of calculation, the graph was represented in three linear functions, and the size of the graph was enlarged by about 2.7 times. The function equation used for the graph is shown below.

The periodic function of the time factor $\mathrm{T}(\mathrm{t})$ was calculated as follows:

$$
\begin{aligned}
& T(t)=\left\{\begin{array}{l}
-0.190819 t+1.38163 \quad t=(2,5.8772) \\
0.008667 t+0.209217 \quad t=(5.8772,21.6186) \\
0.137724 t-2.58088 t=(21.6186,24),[0,2]
\end{array}\right. \\
& T(t+24)=T(t)
\end{aligned}
$$

Where, $t$ means current time and the domain of the definition of $t$ was calculated at the left of each linear function. $T(t)$ indicate a scaling, such that the graph above has a maximum value of one.

\section{Risk Factor 3: Spatial Characteristics}

There are several spatial characteristics that are commonly observed in crime occurrence that can be examined. For example, in some of the cases listed above, the perpetrator spotted the victim in a bright area and committed the crime by chasing the victim after she had moved to a darker area. Factors such as the victim moving from the main road to a narrow alley or moving from a brightly lit area to a dark area can play significant roles in the occurrence of crime. Hence, the following are considered subfactors of the spatial characteristics factor:

a. Decrease in illuminance (moving from a bright place to a dark place) 
b. Decrease in road width detected on the map (moving from a main street to a narrow alley)

c. Existence of video surveillance in the area

d. Accidental crime region

The decrease-in-illuminance value was defined as follows:

$$
l=l_{t}-l_{t-1}
$$

The illuminance of the previous time was subtracted from the current illuminance value (simple difference).

The change-in-road-width value was defined as follows:

$$
r=\left(r_{t}-r_{t-1}\right) / r_{t-1}
$$

The simple difference between the two road widths was calculated and then divided by the original width. By combining the two factors above, the value was calculated based on the equation below. Function $M$ of the spatial characteristics is defined by the $M$ processes receiving the 1 value and the $r$ value.

$$
M(l, r)=\frac{\sum_{l \in T, l<0} l}{\sum_{l \in T} l} \times \frac{\sum_{r \in T, r<0} r}{\sum_{r \in T} r}
$$

The equation above includes an equation for the $l$ value and an equation for the $r$ value. The equation for the $l$ value is the sum of the $l$ values that are reported when the illuminance decreases within the current time slot, divided by the sum of all $l$ values within the current time slot. Hence, this value becomes close to one when the victim moves to a dark area, and it becomes close to zero if the victim moves to a bright area.

The equation for $r$ is the sum of the $r$ values reporting decreases of the road width within the current time slot, divided by the sum of all $r$ values within the current time slot. Hence, this value becomes close to one when the victim moves toward a narrow alley, and it becomes close to zero if the victim moves toward a wide road. Hence, the role played by $\mathrm{M}$ on the different factors of spatial characteristics also has values between $[0,1]$.

Because the three factors all have values between zero and one, the sum of the three factors must have a value between zero and three. When the four sub-factors are considered equivalent to one another, the risk level as influenced by spatial characteristics can be divided into one to four stages.

Hence, the final level of risk can be expressed as follows:

$$
D=T(t)+P(t)+M(l, r)_{t}
$$

$\mathrm{D}=0-1$ : low riskiness

$\mathrm{D}=1-2$ : medium riskiness

$\mathrm{D}=2-3$ : high riskiness

Thus, the real-time risk level for crime occurrence can be calculated using the dynamic factors extracted in Chapter 3 and in real-world situations.

\section{Conclusion}

Dynamic factors of certain crimes must be determined using existing research on crime prevention in order to develop find a scheme for calculating risk associated with each respective factor. By analyzing previous incidents, this study was able to extract potential risk factors for specific crimes and to identify areas with higher risk of crime occurrence 
using the Safety Map application. A scheme for calculating these risk factors in real time was then suggested by analyzing data from 50 criminal cases of roadside kidnapping, rape, and sexual harassment of victims who did not know their attackers.

With the aim of improving public safety and crime prevention, this study attempted to find dynamic factors that may increase or decrease the risk of certain crimes in certain contexts. Static factors based on existing statistical analyses were also examined. The study also attempted to calculate real-time risk levels by analyzing these variable risk factors. Three of these risk factors - floating population, temporal characteristics, and spatial characteristics - were extracted as a result of the analysis, and risk calculation was performed for each factor. Measuring and monitoring real-time risk level in everyday life may be made possible based on the data from this analysis and risk calculation. This study may thus contribute significantly to the implementation of services for preventing crime.

\section{Acknowledgments}

This research was supported by Basic Science Research Program through the National Research Foundation of Korea (NRF) funded by the Ministry of Education(NRF2015R1D1A3A01017968)

\section{References}

[1] P. M. Cozens, G. Saville, \& D. Hillier, "Crime prevention through environmental design (CPTED)", A review and modern bibliography. Property management, vol. 23, no. 5, (2005), pp.328-356.

[2] C. Casteel, \& C. Peek-Asa, "Effectiveness of crime prevention through environmental design (CPTED) in reducing robberies", American Journal of Preventive Medicine, vol. 18, no. 4, (2000), pp.99-115.

[3] R. I. Atlas, "21st century security and CPTED", Designing for critical infrastructure protection and crime prevention, CRC Press, (2013).

[4] R. V. Clarke, "Theoretical Background to crime prevention through environmental design (CPTED) and situational prevention In Designing Out Crime", The Conference Papers, (1989), pp. 13-20.

[5] X. Leiqing, "Research and Practice of CPTED in Architectural Design and Community Planning [J]", New Architecture, vol. 6, no. 1, (2003).

[6] J. M. Tien, T. A. Reppetto, \& L. F. Hanes, "Elements of CPTED”, National Criminal Justice Reference Service. (1977).

[7] M. Rahaman, G.Currie, and C. Muir, "Measuring Station Design Quality for Personal SafetyPreliminary Results", vol. 21.

[8] R. Communities, "Developing \& Maintaining Sustainable Communities", Managing the output focus of Crime Prevention through Environmental Design (CPTED).

[9] G. Owusu, C.Wrigley-Asante, M. Oteng-Ababio, and A.Y. Owusu, "Crime prevention through environmental design (CPTED) and built-environmental manifestations in Accra and Kumasi", Ghana. Crime Prevention \& Community Safety, vol. 17, no. 4, (2015), pp.249-269.

[10] P. Cozens, \& T. Love, "A Review and Current Status of Crime Prevention through Environmental Design (CPTED)", Journal of Planning Literature, (2015).

[11] N. Iranipoor, \& B. Avashpour, "Recognition Of Non-Self-Protected In Residential Areas Based On Addiction Problem", Cumhuriyet Science Journal, vol. 36, no. 4, (2015), pp.1527-1537.

[12] B. Appleyard, C. Ferrell and S. Masthur, "Performance Measurement \& GIS Mapping (A105-A107) A105 The Meaning of "Mean Streets" for Sustainable \& Active Travel", Crime, (Pedestrian/Bike) Casualties and Mode Choice, Journal of Transport \& Health, vol. 2, no. 2, (2015), pp. S59-S60.

[13] Q. Chen, A. Whitbrook, U. Aickelin, and C. Roadknight, "Data classification using the Dempster-Shafer method", Journal of Experimental \& Theoretical Artificial Intelligence, vol. 26, no.4, (2014), pp. 493-517.

[14] D. Suh, and C. Ryu, "A Novel Weighting Method for Context Inference”, International Journal of Multimedia and Ubiquitous Engineering, vol.8, no. 1,(2013), pp.91-100.

[15] S. H. Lee, "Unsupervised Image Classification Using Spatial Region Growing Segmentation and Hierarchical Clustering ", Proceedings of the 2001 KSRS Spring Meeting, no. 4, (2001), pp. 25-33.

[16] D. Suh and C. K. Ryu, "Multi-sensor Data Fusion Using Weighting Method based on Event Frequency", The Journal of The Korea Institute of Electronic Communication Sciences, vol.6, no. 4, (2011), pp. 581-587.

[17] J. Lee, H. Park and S. Oh, "A Study on the Crime Prevention through Environmental Design (CPTED) Strategy for the Application in Korea: A Preliminary Inquiry", Journal of The Korean Regional Development Association, vol. 20, no. 2, (2008), pp. 213-232. 
[18] D. B. Cornish, and R. V. Clarke, "Theory for Practice in Situational Crime Prevention", Crime Prevention Studies, NY: Criminal Justice Pres., (2003).

[19] S. Yoon, C. Ryu, and D. Suh, "A Novel Way of BPA Calculation for Context Inference using Sensor Signals", International Journal of Smart Home, vol. 8, no. 1, (2014), pp. 1-8.

[20] T. Lu, X. Guo, L. Zhao, Y. Li, P. Lin and B. Fang, "Security Model for Sensitive Information Systems and Its Applications in Sensor Networks", IJSIA, vol. 9, no.5, (2015), pp.1-18,

[21] S. Yoon and C. K. Ryu, "Clustering for Context Inference in the Data Stream Mining", IJSEIA, vol. 9, no.1, (2015), pp.105-112.

[22] A. Wang, J. Zhao, Y. Iwahori and S. Dai, "Medical Image Fusion in NSCT Domain Combining with Compressive Sensing", IJMUE, vol.10, no.5, (2015), pp. 1-8.

[23] X. Wang, D. Meng and Y. Xu, "Efficiency Optimization Control for Three-Phase Induction Motorswith Hall Sensor", IJCA, vol. 8, no. 1, (2015), pp.1-8.

[24] A. Elzamly, B. Hussin, S. S. Abu Naser, and M. Doheir, "Classification of Software Risks with Discriminant Analysis Techniques in Software planning Development Process", IJAST, vol. 81, (2015), pp.35-48.

\section{Authors}

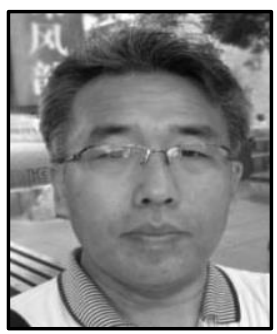

Dong-Hyeok Seoh, He is a professor at Namseoul University. He received the M.S. degrees in computer engineering from Hoseo University in 2005 and the $\mathrm{Ph} . \mathrm{D}$. in computer science from Chungbuk National University in 2012. His research interests included in stream data processing and data fusion in wireless sensor network.

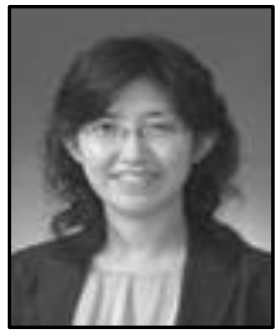

Jeonghwa Song, She is a professor at Namseoul University. She received the B.S. degree in Housing and interior design from Yeonsei University in 1990, and the M.S. and Ph.D. in Housing and interior design from Yeonsei University in 2005. Her research interests are included in data fusion in wireless sensor network and Ubiquitous Housing. 
International Journal of $u-$ and e- Service, Science and Technology Vol.9, No. 1 (2016) 